\title{
Collateral Damage of COVID-19 Pandemic: Delayed Medical Care
}

\author{
Saqib Masroor ${ }^{1}$ \\ ${ }^{1}$ University of Toledo Medical Center
}

April 28, 2020

\begin{abstract}
During the COVID-19 pandemic, ER visits have drastically decreased for non-COVID conditions such as appendicitis, heart attack and stroke. Patients may be avoiding seeking medical attention for fear of catching the deadly condition or as an unintended consequence of stay-at-home orders. This delay in seeking care can lead to increased morbidity and mortality, which has not been figured in the assessment of the extent of damage caused by this pandemic. This case illustrates an example of "collateral damage" caused by COVID-19 pandemic. What would have been a standard STEMI treated with timely and successful stenting of a dominant right coronary artery occlusion, became a much more dangerous post-infarction VSD; all because of a 2-day delay in seeking medical attention by an unsuspecting patient.
\end{abstract}

Collateral Damage of COVID-19 Pandemic: Delayed Medical Care

Saqib Masroor, MD, MHS

University of Toledo College of Medicine and Life Sciences

Heart and Vascular Center

3000 Arlington Avenue

Toledo, OH 43614

Email: saqib.masroor@utoledo.edu

Keywords: COVID-19, Coronary artery disease, Post infarction ventricular septal defect

Short title: "Collateral Damage of COVID-19"

\section{ABSTRACT:}

During the COVID-19 pandemic, ER visits have drastically decreased for non-COVID conditions such as appendicitis, heart attack and stroke. Patients may be avoiding seeking medical attention for fear of catching the deadly condition or as an unintended consequence of stay-at-home orders. This delay in seeking care can lead to increased morbidity and mortality, which has not been figured in the assessment of the extent of damage caused by this pandemic. This case illustrates an example of "collateral damage" caused by COVID19 pandemic. What would have been a standard STEMI treated with timely and successful stenting of a dominant right coronary artery occlusion, became a much more dangerous post-infarction VSD; all because of a 2-day delay in seeking medical attention by an unsuspecting patient.

\section{CASE REPORT:}

A 48 year old male presented to the emergency room (ER) with a 2-day history of persistent chest pain. EKG revealed an ST-elevation myocardial infarction (STEMI) in the inferolateral distribution. Per the ACC guidelines, patient underwent a timely and successful percutaneous revascularization of the dominant right 
coronary artery that was occluded. The left coronary circulation did not have significant disease. A large amount of clot was also removed from the artery during the procedure in which two drug eluting stents were deployed in the mid to distal right coronary artery.

Overnight the patient's condition worsened as he developed cardiogenic shock with tachycardia, hypotension and a loud holo-sytolic murmur was noted. His chest x-ray revealed worsening pulmonary congestion. A stat echocardiogram revealed a large ventricular septal defect (VSD) (Fig 1) and the patient was taken back to the catheterization laboratory for right heart catheterization and placement of intra-aortic balloon pump (IABP). The final diagnosis was a postinfarction VSD measuring $2 \mathrm{~cm}$ in dimension and a shunt fraction of 3. Since the patient's hemodynamics improved with the IABP, we waited a few days to let the myocardium heal somewhat before surgical repair. He also tested negative for COVID-19.

Five days after his hospital presentation, and 7 days after his chest pain started, he was taken to the operating room, where a large $3 \mathrm{~cm}$ VSD was repaired using the exclusion technique with pericardial patch. Except for the upper two 1-1.5 cm, the rest of the septum could not hold any stitches and therefore the pericardial patch was subtended through the anterior wall of the left ventricle, parallel to the left anterior descending artery (interrupted horizontal mattress sutures tied outside the heart on pledgets), through the inferior ventriculotomy (sandwiched between the two layers of muscle closure) and moving up along the lateral wall adjacent to the septolateral junction and extending up to the origin of the anterolateral papillary muscle (Interrupted horizontal mattress sutures tied outside the heart over a pledget). The LAA was excluded with an epicardial clip and epicardial bipolar leads were placed on the lateral wall of left ventricle and the right atrium. The origin of the anterolateral papillary muscle was reinforced with a pledgeted stitch through and through the ventricular wall. Intraoperative TEE showed a competent repair with trace residual shunting (Fig 2). The IABP was removed on postoperative day 1 and he was extubated and weaned off inotropes on the second postoperative day. He is still in hospital, but we hope he will continue to make adequate recovery.

On a separate but very important note, in keeping with the policy of "No Visitation" to prevent spread of COVID-19, his wife and family have not seen him since his admission to the hospital, although they did speak to him by phone before surgery and continue to do so now, that he is extubated.

\section{Discussion:}

This case highlights the issues that have been raised before by others regarding the corona virus, stay-athome regulations and demand for emergency care for non-COVID conditions. A recent article published in the Washington Post (1), has reported that physicians in the United States, Spain and UK have all noticed a serious decline in the number of patients coming to the ER not only for typical problems like appendicitis, bowel obstructions, gall bladder problems but also for the more critical heart attacks and strokes. Metzler reports a 40\% decline in the number of admissions for STEMI and non-STEMI in Austria during the COVID-19 pandemic (2). In a soon to be published report by Garcia (3), number of cardiac catheterization laboratory activations for STEMI have been reduced by almost $40 \%$ in 9 high volume centers in the United States.

The reasons for this decline are many and include a fear for catching a deadly virus in the hospital, the instruction to strictly stay in-house, consideration of many heart related symptoms of shortness of breath and chest discomfort by patients to be related to respiratory infections and best dealt with at home etc.

This patient's pain started two days earlier, but he did not seek attention until later. From conversation with him and his wife, they were concerned about the risk of catching the deadly COVID-19 in the hospital. The patient was not a smoker or a known diabetic, and did not have any family history of coronary artery disease. A heart attack was not on their mind but respiratory infection and fear of COVID-19 was the first and foremost concern of theirs. It was not until his chest pain had persisted for two days that he finally came to ER. Had he come when the pain had started, this patient would likely have had an uneventful recovery after treatment of the RCA occlusion per the ACC guidelines. His avoidance of an ER visit in a timely fashion because of the fear of COVID-19, led to the catastrophic complication of STEMI that surgeons rarely see today because of successful results of timely percutaneous revascularization. 
Whatever the reason for this patient's or that of others to delay seeking medical attention during this pandemic, the concern remains that this delay will lead to increased morbidity and mortality in the coming weeks and months. There most certainly are many others like this patient, who have suffered or will suffer a catastrophic complication due to delay in treatment. Metzler posits that if $40 \%$ of people are not seeking care for acute coronary syndromes, and assuming $40 \%$ of those untreated people suffer a significant complication or death (2), the effect of COVID-19 pandemic on non-COVID related health of our population may be even more devastating than COVID-19 itself. And this case just highlights the fact that this assumption may not be too far from the truth.

\section{REFERENCES.}

1. Bernstein, L. and Sellers FS. (2020) Patients with heart attacks, strokes and even appendicitis vanish from hsoptals. Washington Post. April 19, 2020. https://www.washingtonpost.com/health/patientswith-heart-attacks-strokes-and-even-appendicitis-vanish-from-hospitals/2020/04/19/9ca3ef24-7eb4-11ea9040-68981f488eed_story.html

2. Metzler B, Siostrzonek P, Binder RK, Bauer A and Reinstadler SJ. (2020) Decline of acute coronary syndrome admissions in Austria since the outbreak of COVID-19: the pandemic response causes cardiac collateral damage. Eur Heart J 0, 1-2. Doi:10.1093/eurheartj/ehaa314

3. Garcia S, Albaghdadi MS, Meraj PM, et al. (2020) Reduction in ST-segment elevation cardiac catheterization laboratory activations in the United States during COVID-19 pandemic. J Amer Coll Cardiol, https://doi.org/10.1016/j.jacc.2020.04.011

Fig 1

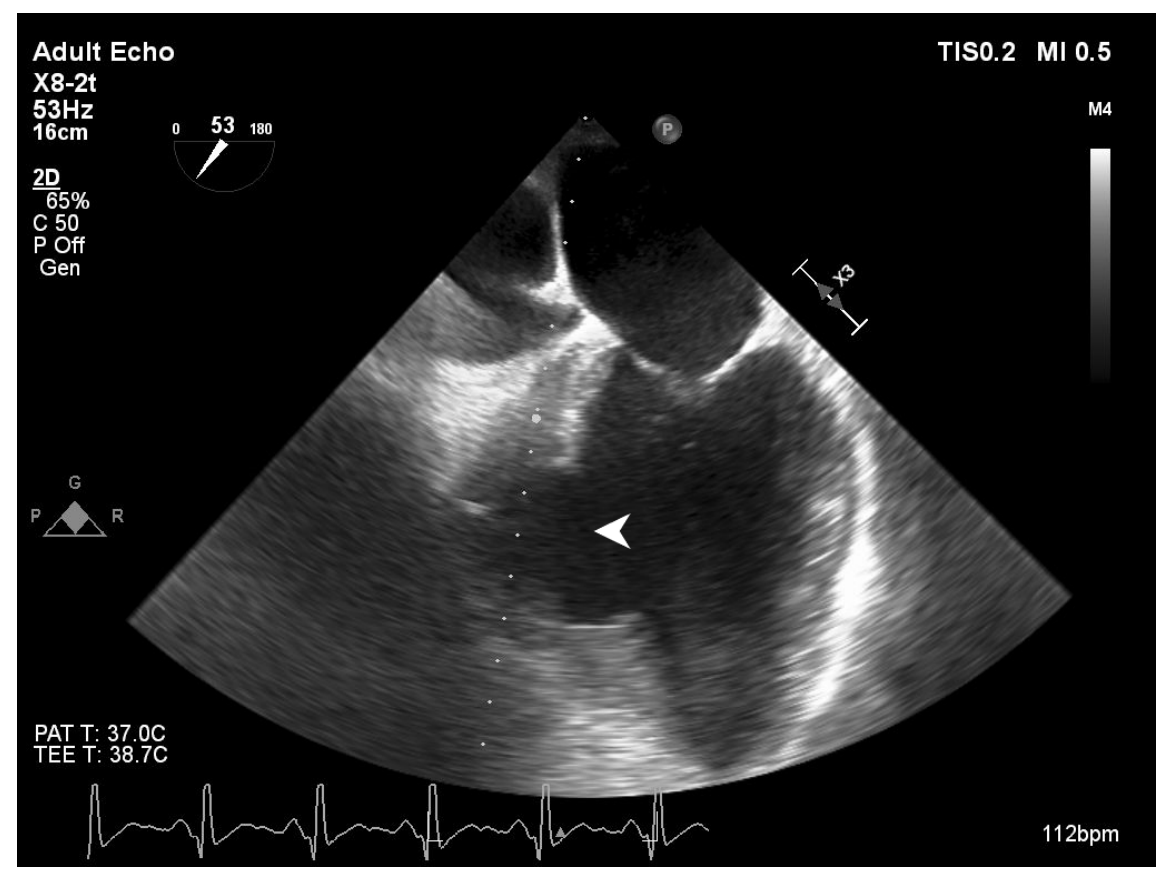

Fig 2. 


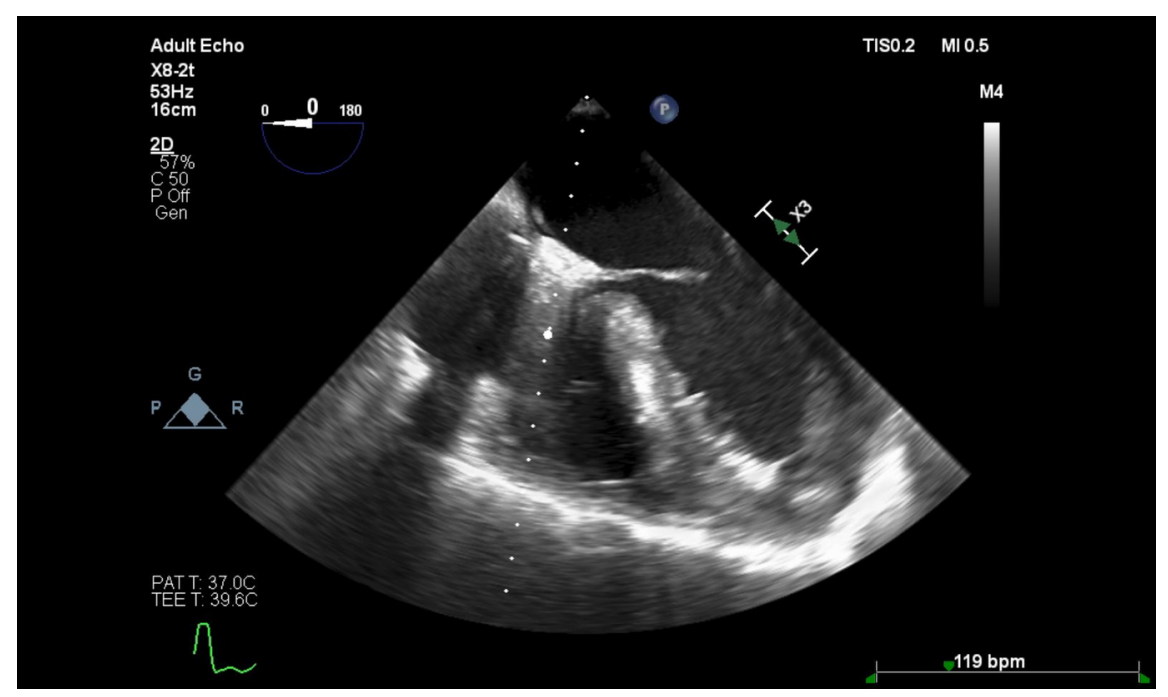

\section{Figure Legend}

FIG 1. Transthoracic echocardiography revealing a large VSD (white arrow) extending form mid septum to the apex.

Fig 2. Intraoperative post-repair transesophageal echocardiography demonstrating a competent repair of the VSD.

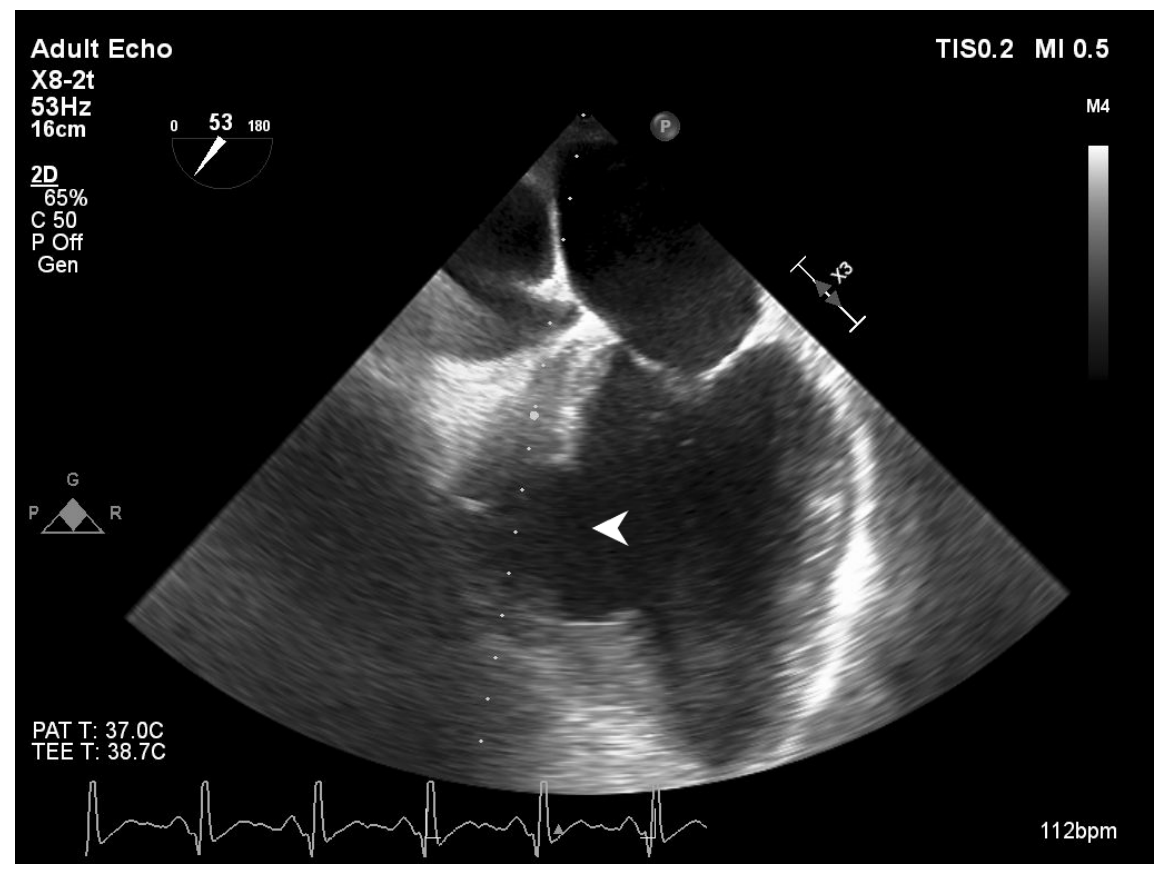




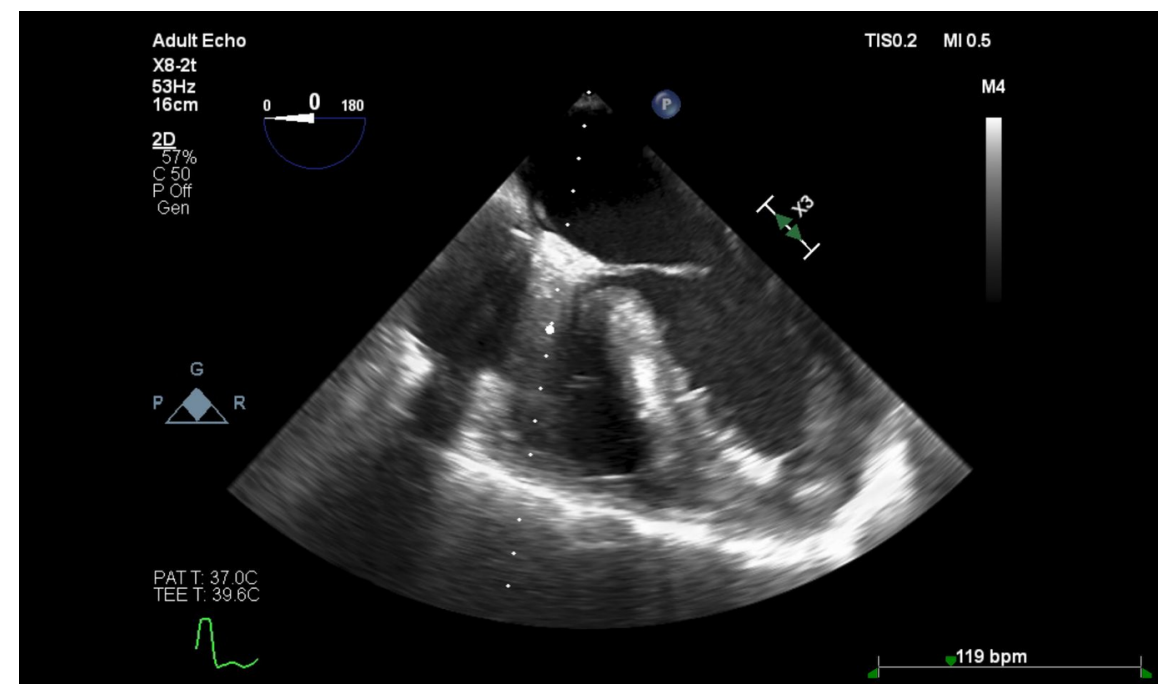

